\title{
Near-Field Direct Antenna Modulation (NFDAM) Transmitter at 2.4GHz
}

\author{
Arthur H. Chang, Aydin Babakhani*, and Ali Hajimiri \\ California Institute of Technology \\ E-mail: ahchang@caltech.edu
}

\section{Introduction}

In direct or two-step up-conversion transmitter architectures, the in-phase and quadrature components of the signal are modulated at base-band, then upconverted to a radio frequency. The resulting modulated signal at the carrier frequency will then need to be amplified and finally transmitted through an antenna. In these systems, antenna patterns remain unchanged at each symbol transmission, and hence receivers located in different directions in space capture the same modulated signal with only differences in power level and time delay. In directional transmitters, while a large portion of the power is coupled to the main beam, a small part is leaked to the side lobes. Thus, even in such systems, a sensitive receiver can eavesdrop on the information by picking up the signal from the side lobes of the antenna. To avoid undesired receivers from capturing the same information, there is a need for a system with the capability of transmitting information in a direction-dependent fashion. As presented and demonstrated in [1][2], Near-Field Direct Antenna Modulation (NFDAM) technique can be used to overcome the security challenge by using direction-dependent information transmission. In these systems, switches or varactors are used to constantly change the scattering properties of antenna reflectors. By varying the boundary conditions of an antenna at the speed of symbol-rate, it is possible to modulate the signal after the antenna as shown in Fig. 1. Because the signal is modulated after the antenna and the scattering properties of the reflectors vary with angle, the receivers located in different directions observe direction-dependent constellation points. In this paper, we demonstrate the feasibility of a NFDAM transmitter at $2.4 \mathrm{GHz}$ on a microstrip setting.

\section{Concept of Near-Field Direct Antenna Modulation}

In our work, a dipole antenna, two reconfigurable reflectors, and nineteen MicroElectro-Mechanical Systems (MEMS) switches are used to form a NFDAM system. The system layout is shown in Fig. 2. The antenna is driven by a CW signal of constant amplitude and phase, and the reflectors can be shorted or opened at some points along its length using MEMS switches. The reflected signal in any given direction then interferes with the main signal radiated by the antenna in that direction. Since the amplitude and phase of the reflected signal depend on the boundary conditions that the reflectors impose and can be varied by toggling the switches, the overall signal received in the far-field can effectively be modulated by changing the states of the switches. 
In NFDAM systems, as the modulated signal does not pass through a power amplifier, narrowband highly-efficient power amplifiers can be used to amplify the carrier signal without disturbing the constellation points. In addition, our system can exhibit a large number of degrees of freedom (namely $2^{\mathrm{N}}$ with $\mathrm{N}$ switches). This allows a broad range of modulation schemes and extra security. It is noteworthy to mention that any arbitrary digital modulation scheme including the non-constant envelope ones can be adopted by this system. Moreover, it will be shown later in this paper that information can be transmitted in a directiondependent fashion by generating the correct signal constellation only in a certain direction and having the constellation graph scrambled in other directions.

\section{Details of Design and Measurement}

In our design, a dipole antenna is used with a wideband microstrip to co-planar waveguide transition [3]. Due to fabrication cost and constraints, we have chosen a Rogers TMM 10i substrate with a dielectric constant of 10.2 to minimize the circuit size. A block diagram of the measurement setup is shown in Fig. 3 along with a picture of the setup in Fig. 4. In the first measurement, the radiated far-field signal from the NFDAM transmitter is characterized using an HP 8753D network analyzer. A LabVIEW program controls the state of the MEMS switches through a data acquisition unit and a control logic PCB. The data acquisition unit sends serial data stream to the control logic PCB and the PCB then generates the voltage swings needed to set the state of the MEMS switches. After setting the desired state for the switches, the LabVIEW program communicates with the network analyzer through a GPIB card. One of the ports of the network analyzer sends a $2.4 \mathrm{GHz}$ signal to the NFDAM transmitter while the other port uses a Tecom Log Periodic antenna at the receiving side to accurately measure the phase and amplitude of the $S_{21}$.

With the antennas in an anechoic chamber, we can accurately measure the phase and amplitude of the $S_{21}$ for each switching combination. The measured real and imaginary parts of the $S_{21}$ can then be plotted on the signal constellation diagram as shown in Fig. 5. In this figure, we have measured the variation of $S_{21}$ in two different directions with an angular separation of approximately 45 degrees (boresight and $\varphi=0^{\circ}, \theta=45^{\circ}$ ) for the same set of switching combinations. We have measured a total of 10,000 randomly chosen switching combinations and selected the ones which result in 20 equally spaced constellation points in the desired boresight direction. As shown in Fig. 5, the signal constellation points are completely scrambled in the undesired direction, proving the feasibility of our system.

Lastly, it is important to measure the return loss $\left(\mathrm{S}_{11}\right)$ of the radiating antenna with different switching combinations to ensure that the impedance variation at the input of the antenna is minimal. With the 10,000 randomly chosen switching combinations, we have measured the $S_{11}$ to have a mean of $-9.98 \mathrm{~dB}$, a maximum of $-9.60 \mathrm{~dB}$, and a minimum of $-10.34 \mathrm{~dB}$. Thus, we have verified that we do not change the antenna input impedance excessively. 


\section{Conclusion}

A Near-Field Direct Antenna Modulation (NFDAM) transmitter at $2.4 \mathrm{GHz}$ is demonstrated on a microstrip setting. The transmitter is capable of transmitting information in a direction-dependent fashion by sending the correct signal constellation only in the desired direction while leaving the signal constellation points scrambled in undesired directions. This direction-dependent constellation scrambling nature of the NFDAM systems prevents undesired receivers to correctly demodulate the signal transmitted to a desired receiver at a specific direction.

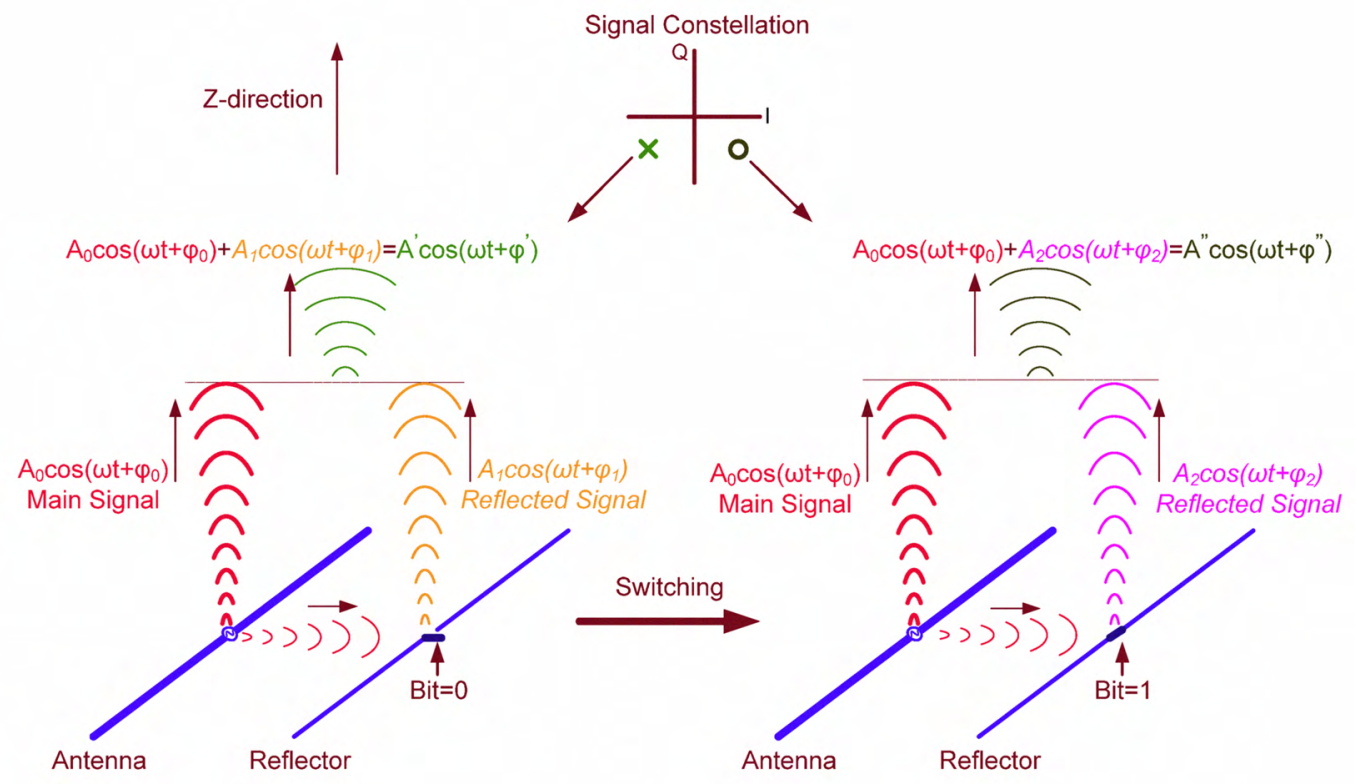

Fig. 1: One-bit modulation in far field by switching the reflector in the near field.

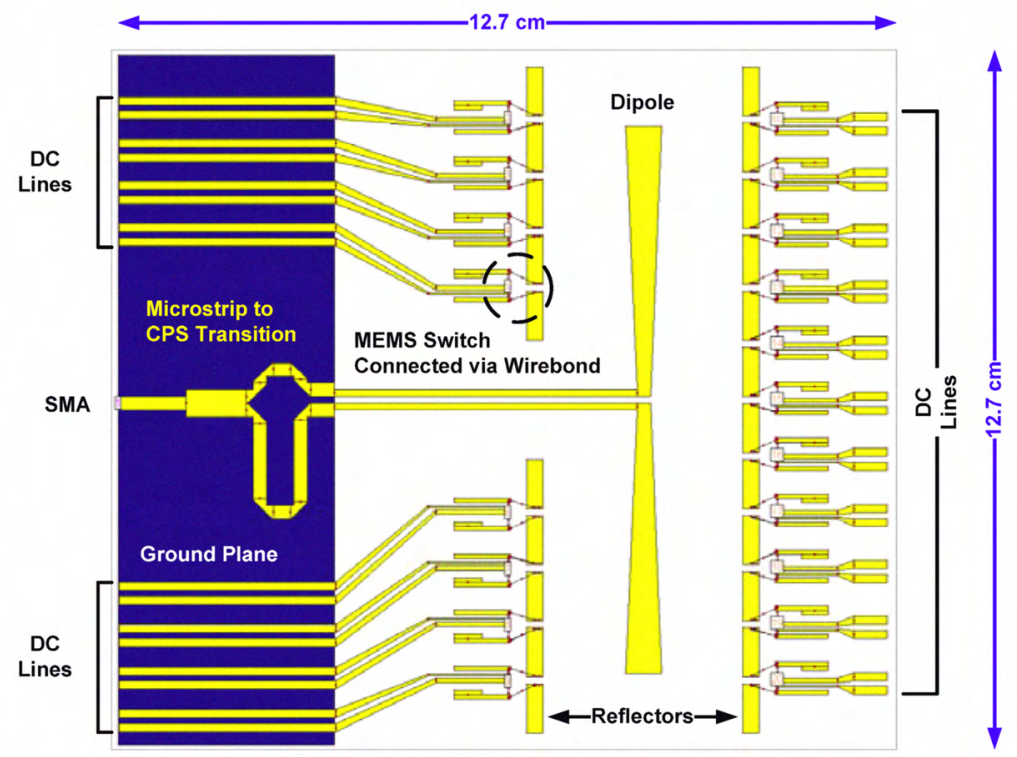

Fig. 2: NFDAM transmitter board layout. 


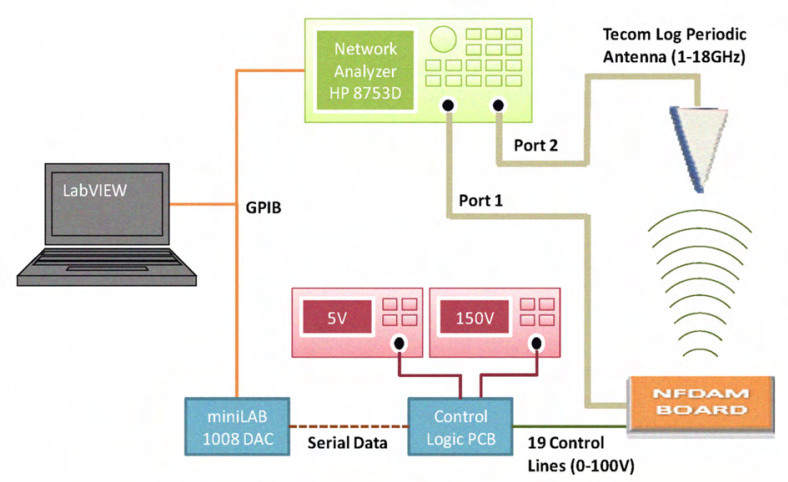

Fig. 3: Measurement setup for the NFDAM characterization.
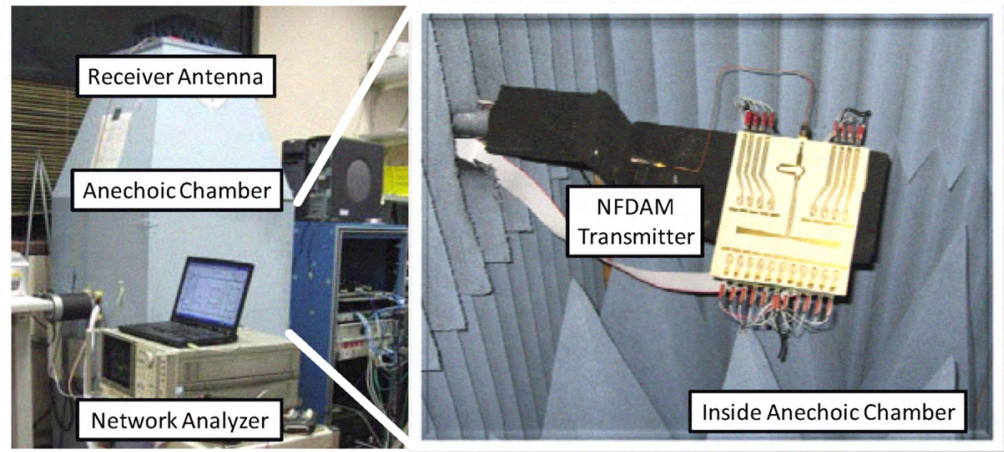

Fig. 4: Picture of the measurement setup.
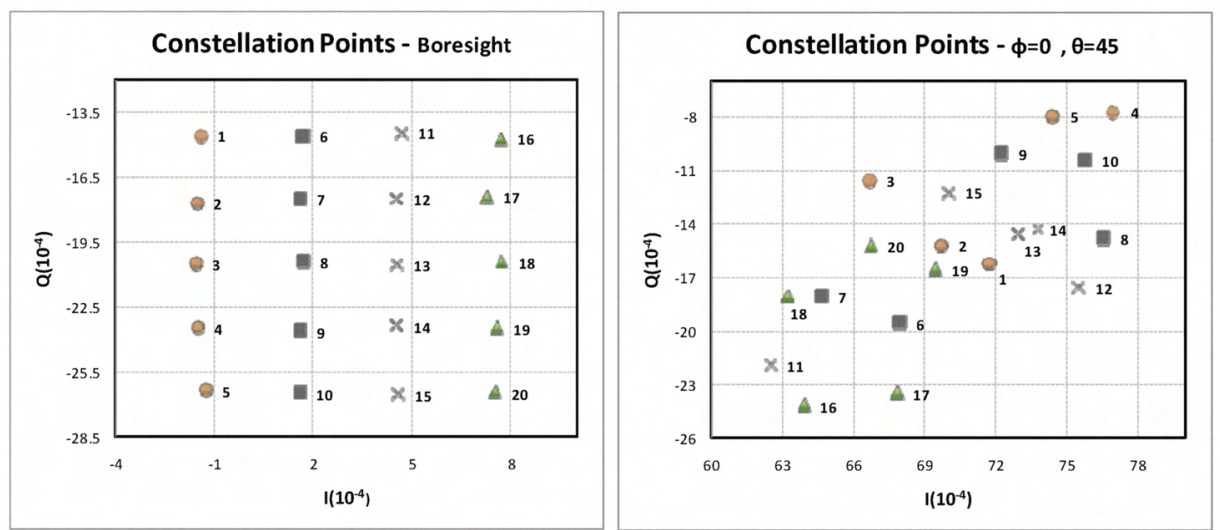

Fig. 5: Measured result of the constellation points of the NFDAM transmitter.

\section{References}

[1] A. Babakhani, D. B. Rutledge, and A. Hajimiri, "A Near-Field Modulation Technique Using Antenna Reflector Switching," in IEEE ISSCC Dig. Tech. Papers, pp.188-189, Feb. 2008.

[2] A. Babakhani, D. B. Rutledge, and A. Hajimiri, "Transmitter Architectures Based on Near-Field Direct Antenna Modulation (NFDAM)", in IEEE $J$. Solid-State Circuits, vol. 43, no. 12, pp. 2674-2692, Dec. 2008.

[3] M. Abdalla, S. Salous. "Uniplanar wideband quasi-Yagi antenna for multiple antenna channel measurements," Proc. London Comm. Symp., Sept. 2002. 